\title{
MODIFICATION OF THE AMPLITUDE OF THE HUMAN ELECTRO-OCULOGRAM BY LIGHT AND DARK ADAPTATION*
}

BY

\section{J. FRANÇOIS, G. VERRIEST, AND A. DE ROUCK \\ From the Ophthalmological Clinic of the University of Ghent, Belgium. Director: Prof. Dr. J. François}

WHEN an electrode is placed on the cornea and another near the posterior pole of the eye (or elsewhere on the body) a standing potential is found (Kohlrausch, 1931; Marg, 1951). In vertebrates the cornea is positive in relation to the retina. The axis of this electrical system is approximately the same as the optic axis. If electrodes are placed near the eye and if the eye is turned, the electrode closest to the positive corneal pole will be positive in relation to the others. This recorded electro-oculographic response (EOG) depends on the angle and speed at which the globe rotates; it is independent of the action currents of the eye muscles, which are not recorded.

Fig. 1 shows a normal human EOG; the electrodes are placed round the left eye, and when the patient looks to the right (MR) this eye is adducted and electrode (1) on the nasal side becomes nearer to the cornea and positive to electrode (3) on the temporal side; we obtain also an upward deflexion on the derivation $1 / 3$.
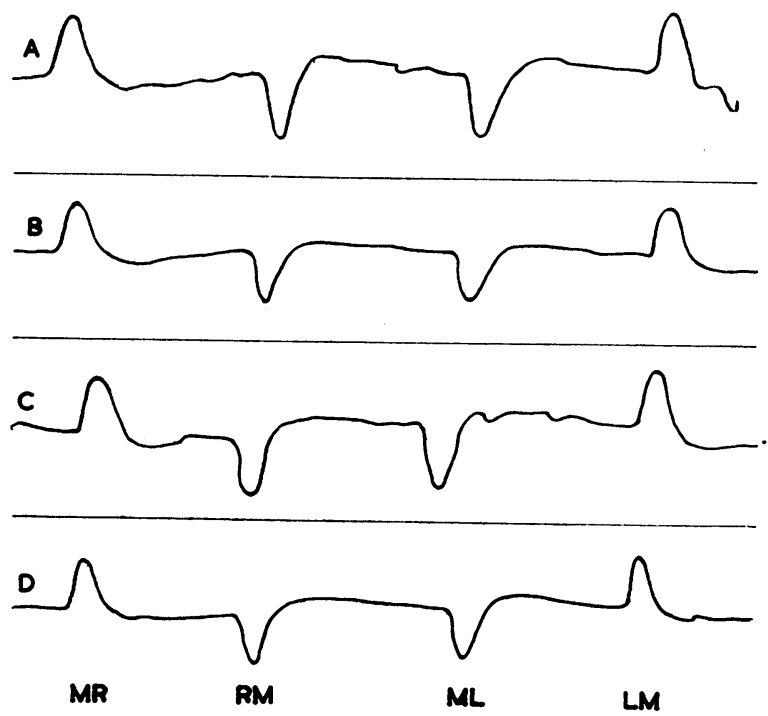

Fig. 1.-Electro-oculogram of Subject 2. Derivation 1/3.
A. Before light adaptation.
C. At the beginning of dark adaptation (increased).
B. At the end of light adaptation (reduced).
D. At the end of dark adaptation (again reduced).

* Received for publication February 21, 1955. 
The only communication relating to the modification of the human EOG by light and dark adaptation is that of Miles (1940). According to this author, there is a statistically valid augmentation of $0.38 \mathrm{mV}$ when the illumination of the subject varies from 0.001 to $5 \mathrm{~mL} \dagger$; each time the subject is submitted to a new illumination the amplitude of the EOG is modified for about 5 minutes and especially during the last 2 minutes.

We have, for two reasons, made a new study of the eventual relation between the amplitude of the EOG and the state of adaptation: first, because Miles's results are not in agreement with those of Wulff and Freyburger (1948) using animals (according to these authors, the standing potential of the excised eye of a frog augments during dark adaptation, as does the $b$-wave of the ERG), and secondly, because Miles based his experiments on levels of illumination and not on thresholds of perception.

\section{Technique}

Light and Dark Adaptation.-We used the Goldmann-Weekers adaptometer (2nd model). This apparatus consists chiefly of a hemisphere corresponding to the whole binocular visual field of the subject, who may thus be exposed to a global light adaptation which may be varied from 1,000 to $8,000 \mathrm{asb}$.

During dark adaptation, we determined the global thresholds for white light (integral adaptometry with contrast only in time; duration of the bright and dark phases: 1 sec.).

Electro-Oculography.-We used non-polarizable silver electrodes attached to the skin with collodion. The skin was cleansed with ether and contact was aided with commercial electrode jelly. Three electrodes were placed round the left eye:

(1) on the base of the nose near the inner canthus,

(2) on the inferior orbital margin, a little to the nasal side of the sagittal plane of the eye,

(3) on the temporal orbital margin, near the outer canthus.

A distant electrode (4) was placed on the occiput. At different stages of the experiments, the subject performed four successive ocular movements, as rapidly as possible:

(i) from the middle to the extreme right (MR),

(ii) from the extreme right to the middle (RM),

(iii) from the middle to the extreme left (ML),

(iv) from the extreme left to the middle (LM).

We used this technique in preference to limited excursions (e.g. $\left.30^{\circ}\right)$ because the results are more constant. As a control these movements were performed in an uninterrupted series. An ink-electroencephalograph was used for the registration.

Experiments.-With each subject we first took an EOG in moderate brightness, without pre-adaptation to dark or light (WA). Subjects 1, 2, and 3 were then light-adapted (2,000 asb. for $5 \mathrm{~min}$.) and the eye movements were recorded immediately after the fifth minute (L 1). The subjects were subsequently darkadapted under adaptometric control and the EOG was recorded at different levels

$\dagger 1$ apostilb $(\mathrm{asb})=1.010^{-1}$ millilamberts $(\mathrm{mL})=\frac{1}{\pi} 10^{-4} \mathrm{stilb}\left(\mathrm{sb}, 1 \mathrm{~cd} / \mathrm{cm}^{2}\right)$, if luminance and brilliance may be confused. 
of adaptation, referred to in the Tables in $\log$ asb (D 2). The subjects were then again light-adapted and a new EOG was recorded (L 2).

During the examination of Subject 4 we added between WA and L 1 a supplementary recording in moderate dark adaptation without previous light adaptation (D 1); we also followed the course of modification of the EOG for a few minutes after L 2, with Subject 4 in moderate brightness (return to normal: RN). This RN was also tested with Subject 2.

During the examination of Subject 5, the EOG-modification during dark adaptation was measured, first without previous light adaptation (D 1), then after previous light adaptation of $2,000 \mathrm{asb}$. for $5 \mathrm{~min}$. (B 2), and finally after previous dark adaptation of 4,000 asb. for $5 \mathrm{~min}$. (D 3).

\section{Results}

The mean amplitudes of the deflexions of the EOG, all converted into $\mu \mathrm{V}$, are given in Tables $\mathrm{I}$ to $\mathrm{V}$ and in Figs 2 to 7 . The results concerning the "monopolar" derivations $1 / 4$ (nasal) and $3 / 4$ (temporal) and the " bipolar" derivation $1 / 3$ are fully reproduced; we have calculated for each the absolute mean $(m)$ and the fourth of the algebraic sum $(\delta)$ of the deflexions; theoretically $\delta$ must equal zero and the values given indicate errors due to irregularities of conduction and differences of amplitude of the movements. $M$ is the mean of the two $m$ corresponding to the "monopolar" derivations. The infra-orbital derivation $2 / 4$ was only used to control the vertical components of the movements; we have given only the values of MR. The duration of the deflexions remained constant in all conditions and for all the subjects (almost $0.60 \mathrm{sec}$.); the amplitude of the deflexion is thus proportional to the resting potential, disregarding the extra-ocular factors.

\section{Conclusions}

(1) Light adaptation (2,000 asb, $5 \mathrm{~min}$.) gives a decrease of amplitude of the electro-oculographic deflexions; after the end of a period in the dark, the deflexions become larger, so that the values are soon greater than before the light adaptation.

(2) Dark adaptation after light adaptation is characterized by a fall in the EOG-amplitudes, which begins immediately after the rise which follows the end of the light adaptation; it is very rapid at first and becomes progressively slower. At the end of the dark adaptation the EOG remains stationary or rises slightly, even if the perception threshold continues to decrease. One of the subjects was light-adapted successively at 2,000 and 4,000 asb., and the modifications of the EOG were the same after these two different pre-adaptations.

(3) If the subject was dark-adapted without preliminary light adaptation, the amplitude of the EOG-deflexions for a given threshold was markedly higher than when the subject was in the dark.

(4) In contrast to the $b$-wave of the ERG, there is no constant relation between the thresholds of perception and the relative amplitudes of the 


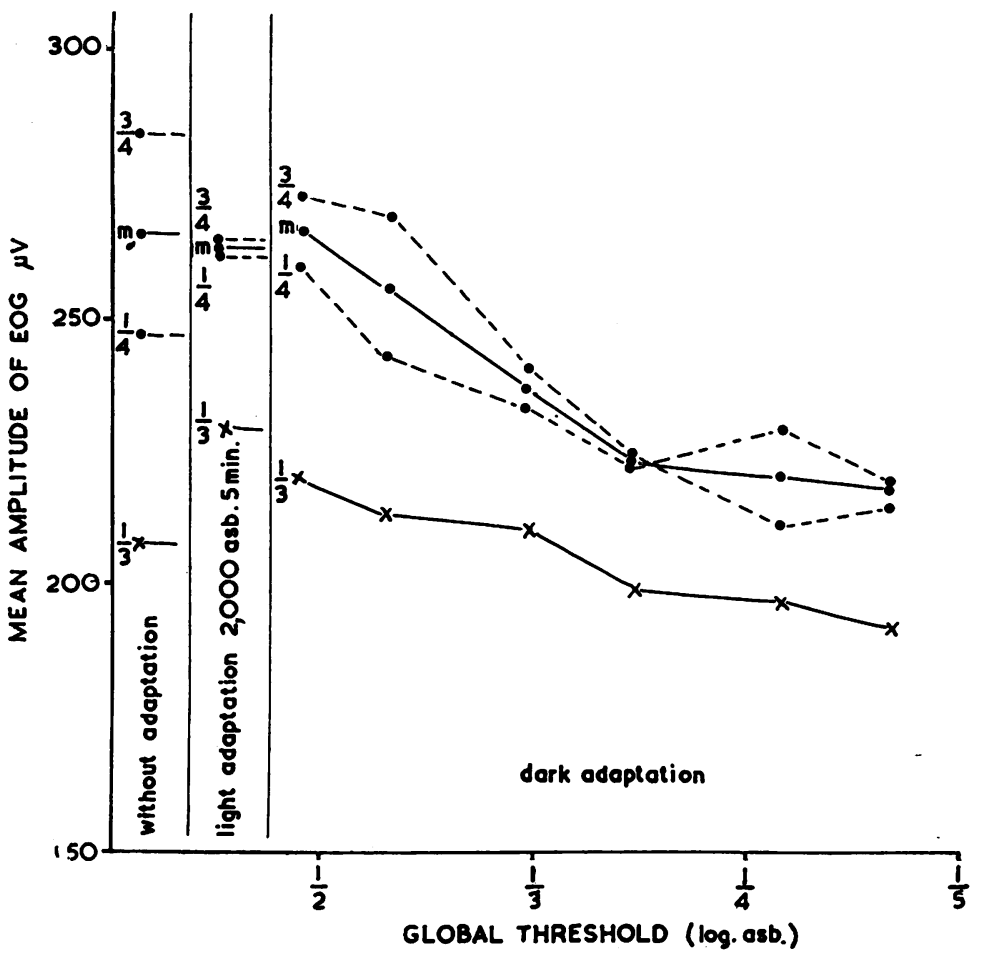

Fig. 2.-Subject 1.

TABLE I

SUBJECT 1

\begin{tabular}{|c|c|c|c|c|c|c|c|c|c|}
\hline & & \multirow[t]{2}{*}{ W A } & \multirow[t]{2}{*}{ L 1} & \multicolumn{6}{|c|}{ D 2} \\
\hline & & & & $2.3-3.9$ & $3.8-3.6$ & $3.1-3.0$ & $4 \cdot 6-4 \cdot 5$ & $5.9-5.7$ & $5 \cdot 3$ \\
\hline $1 / 4$ & $\begin{array}{l}\text { MR } \\
\mathbf{R M} \\
\mathbf{M L} \\
\mathbf{L M} \\
\text { mean } \\
\delta\end{array}$ & $\begin{array}{r}+294 \\
-255 \\
-260 \\
+305 \\
286 \\
+\quad 21\end{array}$ & $\begin{array}{r}280 \\
235 \\
269 \\
269 \\
263 \\
+11\end{array}$ & $\begin{array}{r}294 \\
286 \\
224 \\
280 \\
273 \\
+16\end{array}$ & $\begin{array}{r}302 \\
252 \\
238 \\
286 \\
269 \\
+24\end{array}$ & $\begin{array}{r}277 \\
244 \\
160 \\
280 \\
240 \\
+40\end{array}$ & $\begin{array}{r}269 \\
224 \\
148 \\
258 \\
225 \\
+40\end{array}$ & $\begin{array}{r}263 \\
218 \\
117 \\
249 \\
212 \\
+49\end{array}$ & $\begin{array}{r}269 \\
190 \\
140 \\
266 \\
216 \\
+51\end{array}$ \\
\hline $3 / 4$ & $\begin{array}{l}\text { MR } \\
\mathbf{R M} \\
\mathbf{M} \mathbf{L} \\
\mathbf{L M} \\
\text { mean } \\
\delta \\
\end{array}$ & $\begin{array}{r}-235 \\
+242 \\
+262 \\
-253 \\
248 \\
+\quad 4\end{array}$ & $\begin{array}{r}255 \\
262 \\
301 \\
260 \\
264 \\
+14 \\
\end{array}$ & $\begin{array}{l}255 \\
255 \\
279 \\
253 \\
261 \\
+6\end{array}$ & $\begin{array}{r}207 \\
249 \\
268 \\
249 \\
243 \\
+13 \\
\end{array}$ & $\begin{array}{r}211 \\
246 \\
242 \\
238 \\
234 \\
+10 \\
\end{array}$ & $\begin{array}{r}187 \\
229 \\
242 \\
231 \\
222 \\
+13 \\
\end{array}$ & $\begin{array}{r}198 \\
246 \\
246 \\
229 \\
230 \\
+17 \\
\end{array}$ & $\begin{array}{r}176 \\
220 \\
251 \\
227 \\
218 \\
+69 \\
\end{array}$ \\
\hline$\frac{1 / 4+3 / 4}{2}$ & $\delta^{M}$ & $\begin{array}{r}267 \\
17\end{array}$ & $\begin{array}{r}264 \\
12\end{array}$ & $\begin{array}{r}267 \\
10\end{array}$ & $\begin{array}{r}256 \\
11\end{array}$ & $\begin{array}{r}237 \\
30\end{array}$ & $\begin{array}{r}223 \\
27\end{array}$ & $\begin{array}{r}221 \\
32\end{array}$ & $\begin{array}{r}217 \\
33\end{array}$ \\
\hline $1 / 3$ & $\begin{array}{l}\text { MR } \\
\mathbf{R M} \\
\mathbf{M L} \\
\mathbf{L M} \\
\text { mean } \\
\delta\end{array}$ & $\begin{array}{r}+251 \\
-187 \\
-207 \\
+189 \\
208 \\
+11\end{array}$ & $\begin{array}{r}238 \\
205 \\
257 \\
220 \\
230 \\
+1\end{array}$ & $\begin{array}{l}220 \\
202 \\
251 \\
213 \\
222 \\
-5\end{array}$ & $\begin{array}{l}216 \\
191 \\
249 \\
198 \\
213 \\
-6\end{array}$ & $\begin{array}{l}202 \\
196 \\
235 \\
207 \\
210 \\
-5\end{array}$ & $\begin{array}{l}185 \\
185 \\
220 \\
205 \\
199 \\
-4\end{array}$ & $\begin{array}{l}180 \\
180 \\
231 \\
196 \\
197 \\
-9\end{array}$ & $\begin{array}{r}169 \\
174 \\
233 \\
194 \\
192 \\
-11\end{array}$ \\
\hline
\end{tabular}




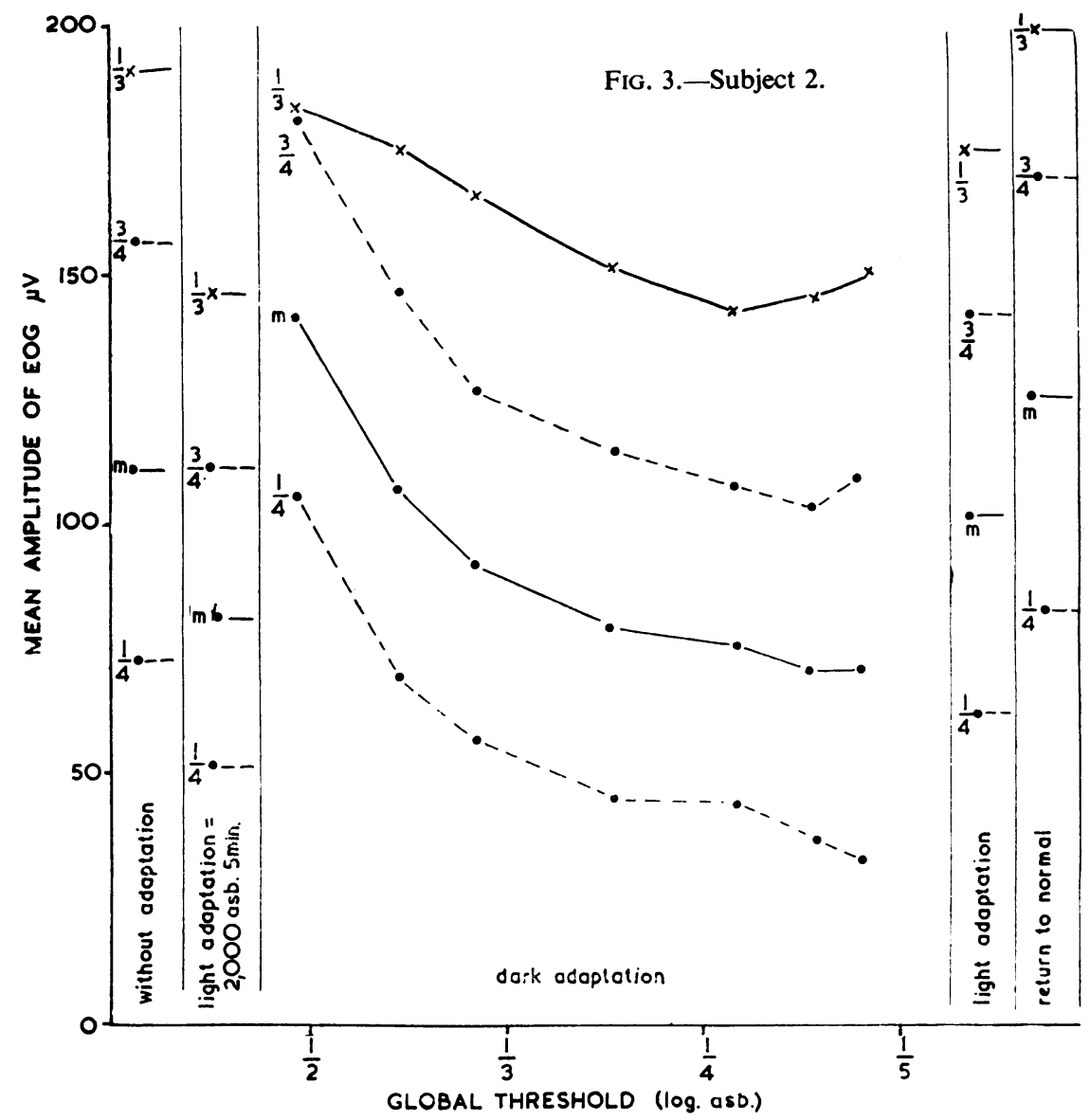

TABLE II

SUBJECT 2

\begin{tabular}{|c|c|c|c|c|c|c|c|c|c|c|c|c|}
\hline & & \multirow[t]{2}{*}{ W A } & \multirow[t]{2}{*}{ L 1} & \multicolumn{7}{|c|}{ D 2} & \multirow[t]{2}{*}{ L 2} & \multirow[t]{2}{*}{$\mathbf{R} \mathbf{N}$} \\
\hline & & & & $\begin{array}{l}2 \cdot 5 \\
-3 \cdot 8 \\
\end{array}$ & $\begin{array}{r}3.6 \\
-3.5 \\
\end{array}$ & $\begin{array}{l}3 \cdot 3 \\
-3 \cdot 0 \\
\end{array}$ & $\begin{array}{l}4 \cdot 7 \\
-4 \cdot 2 \\
\end{array}$ & $\begin{array}{l}5.9 \\
-5.7 \\
\end{array}$ & $\begin{array}{l}5 \cdot 6 \\
-5 \cdot 3 \\
\end{array}$ & $\begin{array}{l}5 \cdot 3 \\
-5 \cdot 2 \\
\end{array}$ & & \\
\hline $1 / 4$ & $\begin{array}{l}\text { MR } \\
\text { RM } \\
\text { ML } \\
\text { LM } \\
\text { mean } \\
\delta \\
\end{array}$ & $\begin{array}{l}+80 \\
-60 \\
-32 \\
+116 \\
72 \\
+26 \\
\end{array}$ & $\begin{array}{r}45 \\
36 \\
43 \\
81 \\
51 \\
+12 \\
\end{array}$ & $\begin{array}{r}131 \\
108 \\
66 \\
116 \\
105 \\
+18 \\
\end{array}$ & $\begin{array}{r}71 \\
93 \\
33 \\
76 \\
69 \\
+5 \\
\end{array}$ & $\begin{array}{r}72 \\
53 \\
25 \\
81 \\
57 \\
+18 \\
\end{array}$ & $\begin{array}{r}55 \\
45 \\
25 \\
55 \\
45 \\
+10 \\
\end{array}$ & $\begin{array}{r}60 \\
41 \\
8 \\
65 \\
43 \\
+18 \\
\end{array}$ & $\begin{array}{r}55 \\
46 \\
0 \\
45 \\
36 \\
+14 \\
\end{array}$ & $\begin{array}{r}63 \\
33 \\
0 \\
33 \\
32 \\
+16 \\
\end{array}$ & $\begin{array}{r}73 \\
58 \\
33 \\
33 \\
61 \\
+4 \\
\end{array}$ & $\begin{array}{r}99 \\
73 \\
41 \\
116 \\
82 \\
+25 \\
\end{array}$ \\
\hline $3 / 4$ & $\begin{array}{l}\text { MR } \\
\text { RM } \\
\text { ML } \\
\text { LM } \\
\text { mean } \\
\delta \\
\end{array}$ & $\begin{array}{r}-130 \\
+166 \\
+166 \\
-156 \\
155 \\
+38 \\
\end{array}$ & $\begin{array}{r}85 \\
158 \\
100 \\
103 \\
111 \\
+17 \\
\end{array}$ & $\begin{array}{r}154 \\
201 \\
201 \\
.164 \\
180 \\
+21 \\
\end{array}$ & $\begin{array}{l}100 \\
183 \\
154 \\
149 \\
146 \\
+6 \\
\end{array}$ & $\begin{array}{r}75 \\
153 \\
149 \\
129 \\
127 \\
+24 \\
\end{array}$ & $\begin{array}{r}66 \\
140 \\
103 \\
149 \\
114 \\
+7 \\
\end{array}$ & $\begin{array}{r}65 \\
133 \\
111 \\
120 \\
107 \\
+15 \\
\end{array}$ & $\begin{array}{r}55 \\
133 \\
116 \\
110 \\
103 \\
+21 \\
\end{array}$ & $\begin{array}{r}66 \\
123 \\
123 \\
123 \\
109 \\
+14 \\
\end{array}$ & $\begin{array}{r}160 \\
171 \\
147 \\
146 \\
141 \\
+3 \\
\end{array}$ & $\begin{array}{r}149 \\
183 \\
188 \\
159 \\
170 \\
+15 \\
\end{array}$ \\
\hline$\frac{1 / 4+3 / 4}{2}$ & $\delta^{M}$ & $\begin{array}{r}113 \\
12\end{array}$ & 81 & $\begin{array}{r}142 \\
3\end{array}$ & $\begin{array}{r}108 \\
1\end{array}$ & $\begin{array}{r}92 \\
6\end{array}$ & $\begin{array}{r}80 \\
3\end{array}$ & $\begin{array}{r}75 \\
3\end{array}$ & $\begin{array}{r}70 \\
7\end{array}$ & $\begin{array}{r}70 \\
2 \\
\end{array}$ & $\begin{array}{r}101 \\
1\end{array}$ & $\begin{array}{r}125 \\
10\end{array}$ \\
\hline $1 / 3$ & $\begin{array}{l}\text { MR } \\
\text { RM } \\
\text { ML } \\
\mathbf{L M} \\
\text { mean } \\
\delta \\
\end{array}$ & $\begin{array}{r}+198 \\
-196 \\
-196 \\
+173 \\
190 \\
-5 \\
\end{array}$ & $\begin{array}{l}151 \\
149 \\
148 \\
143 \\
146 \\
-1 \\
\end{array}$ & $\begin{array}{l}183 \\
176 \\
194 \\
166 \\
180 \\
-5 \\
\end{array}$ & $\begin{array}{l}168 \\
168 \\
169 \\
166 \\
175 \\
-1 \\
\end{array}$ & $\begin{array}{l}146 \\
172 \\
168 \\
179 \\
166 \\
-4 \\
\end{array}$ & $\begin{array}{r}156 \\
153 \\
143 \\
153 \\
151 \\
+4 \\
\end{array}$ & $\begin{array}{r}143 \\
143 \\
143 \\
143 \\
143 \\
0\end{array}$ & $\begin{array}{l}138 \\
149 \\
148 \\
148 \\
146 \\
-3 \\
\end{array}$ & $\begin{array}{l}143 \\
154 \\
149 \\
167 \\
151 \\
+1\end{array}$ & $\begin{array}{l}166 \\
183 \\
181 \\
166 \\
174 \\
+8\end{array}$ & $\begin{array}{l}212 \\
191 \\
219 \\
171 \\
198 \\
-7\end{array}$ \\
\hline $2 / 4$ & MR & 83 & 81 & 78 & 83 & 78 & 50 & 63 & 63 & 63 & 83 & 90 \\
\hline
\end{tabular}




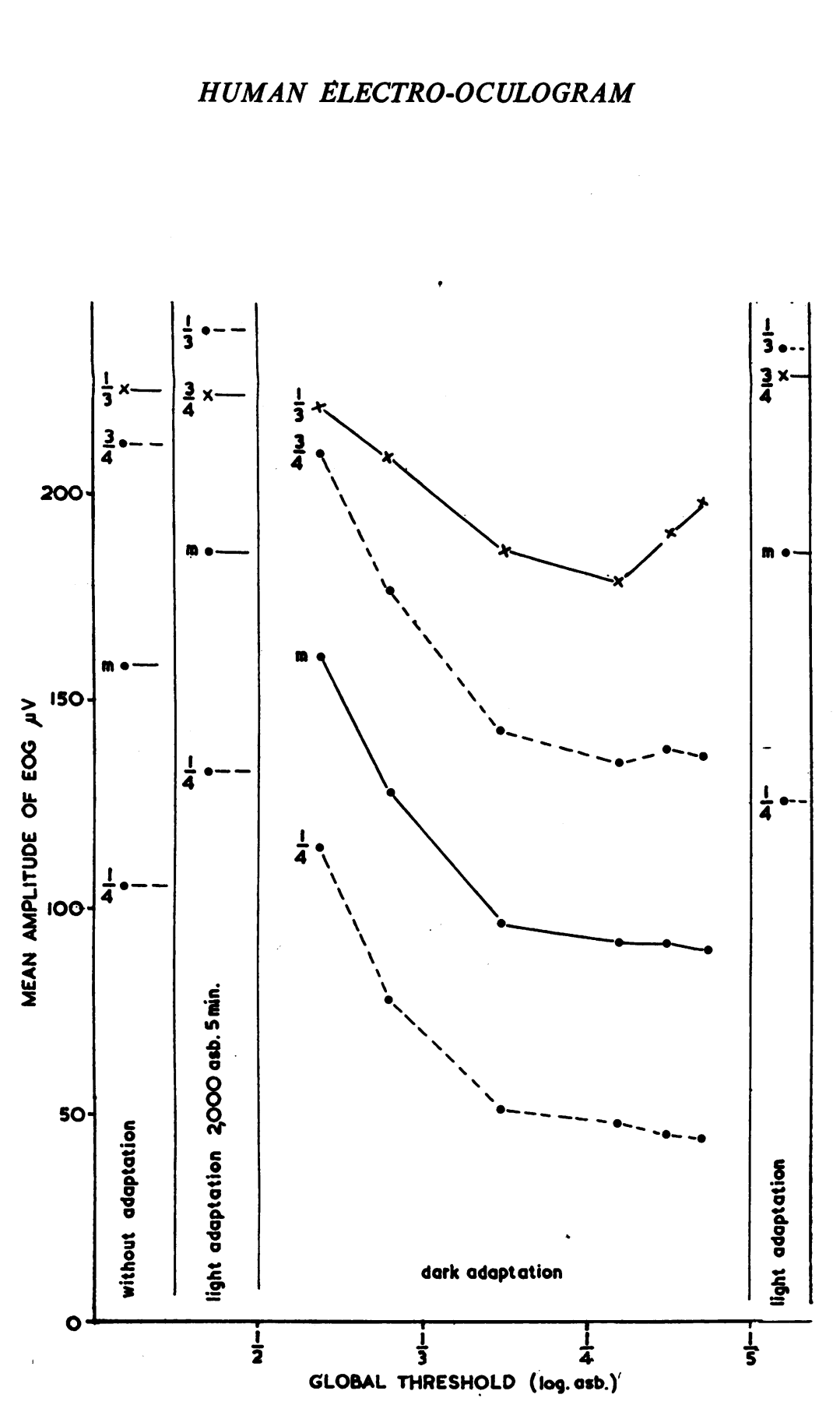

403

Fig. 4.-Subject 3. 
TABLE III

SUBJECT 3

\begin{tabular}{|c|c|c|c|c|c|c|c|c|c|c|}
\hline & & \multirow[t]{2}{*}{ W A } & \multirow[t]{2}{*}{ L 1} & \multicolumn{6}{|c|}{ D 2} & \multirow[t]{2}{*}{ L 2} \\
\hline & & & & $3 \cdot 6$ & $3 \cdot 2$ & $4 \cdot 5$ & $5 \cdot 8$ & $5 \cdot 5$ & $5 \cdot 3$ & \\
\hline $1 / 4$ & $\begin{array}{l}\text { MR } \\
\mathbf{R M} \\
\mathbf{M L} \\
\mathbf{L M} \\
\text { mean } \\
\delta\end{array}$ & $\begin{array}{r}+100 \\
-\quad 72 \\
+\quad 94 \\
+154 \\
105 \\
+22\end{array}$ & $\begin{array}{r}138 \\
40 \\
140 \\
214 \\
133 \\
+43\end{array}$ & $\begin{array}{r}110 \\
54 \\
132 \\
156 \\
113 \\
+20\end{array}$ & $\begin{array}{r}68 \\
28 \\
90 \\
126 \\
78 \\
+19\end{array}$ & $\begin{array}{r}50 \\
0 \\
76 \\
82 \\
52 \\
+14\end{array}$ & $\begin{array}{r}54 \\
0 \\
50 \\
88 \\
48 \\
+23\end{array}$ & $\begin{array}{r}46 \\
22 \\
52 \\
60 \\
45 \\
+8\end{array}$ & $\begin{array}{r}52 \\
16 \\
60 \\
50 \\
44 \\
+6\end{array}$ & $\begin{array}{r}118 \\
84 \\
154 \\
192 \\
137 \\
+18\end{array}$ \\
\hline $3 / 4$ & $\begin{array}{l}\text { MR } \\
\mathbf{R M} \\
\mathbf{M L} \\
\mathbf{L M} \\
\text { mean } \\
\delta\end{array}$ & $\begin{array}{r}-176 \\
+246 \\
+228 \\
-194 \\
211 \\
+26\end{array}$ & $\begin{array}{r}198 \\
254 \\
274 \\
232 \\
239 \\
-25\end{array}$ & $\begin{array}{r}156 \\
236 \\
244 \\
204 \\
210 \\
+30\end{array}$ & $\begin{array}{r}116 \\
194 \\
214 \\
186 \\
177 \\
+26\end{array}$ & $\begin{array}{r}66 \\
143 \\
200 \\
164 \\
143 \\
+28\end{array}$ & $\begin{array}{r}66 \\
124 \\
188 \\
166 \\
136 \\
+20\end{array}$ & $\begin{array}{r}96 \\
128 \\
166 \\
164 \\
138 \\
+8\end{array}$ & $\begin{array}{r}98 \\
152 \\
152 \\
146 \\
137 \\
+15\end{array}$ & $\begin{array}{r}216 \\
240 \\
274 \\
212 \\
235 \\
+21\end{array}$ \\
\hline$\frac{1 / 4+3 / 4}{2}$ & $\delta^{M}$ & $\begin{array}{r}158 \\
4\end{array}$ & $\begin{array}{r}186 \\
18\end{array}$ & $\begin{array}{r}161 \\
10\end{array}$ & $\begin{array}{r}128 \\
7\end{array}$ & $\begin{array}{l}97 \\
14\end{array}$ & $\begin{array}{r}92 \\
3\end{array}$ & $\begin{array}{r}92 \\
0\end{array}$ & $\begin{array}{r}90 \\
9\end{array}$ & $\begin{array}{r}186 \\
3\end{array}$ \\
\hline $1 / 3$ & $\begin{array}{l}\text { MR } \\
\text { RM } \\
\text { ML } \\
\text { LM } \\
\text { mean } \\
\delta\end{array}$ & $\begin{array}{r}+252 \\
-200 \\
-208 \\
+240 \\
225 \\
-\quad 21\end{array}$ & $\begin{array}{r}254 \\
190 \\
222 \\
232 \\
224 \\
+18\end{array}$ & $\begin{array}{r}250 \\
194 \\
206 \\
236 \\
221 \\
+21\end{array}$ & $\begin{array}{r}210 \\
184 \\
204 \\
240 \\
209 \\
+16\end{array}$ & $\begin{array}{l}156 \\
150 \\
210 \\
228 \\
186 \\
+6\end{array}$ & $\begin{array}{r}156 \\
132 \\
192 \\
234 \\
178 \\
+17\end{array}$ & $\begin{array}{r}214 \\
154 \\
194 \\
200 \\
190 \\
+17\end{array}$ & $\begin{array}{r}200 \\
176 \\
192 \\
224 \\
198 \\
+14\end{array}$ & $\begin{array}{r}272 \\
182 \\
228 \\
234 \\
229 \\
+24\end{array}$ \\
\hline $2 / 4$ & RM & 134 & 152 & 158 & 112 & 76 & 72 & 86 & 78 & 204 \\
\hline
\end{tabular}

TABLE IV

SUBJECT 4

\begin{tabular}{|c|c|c|c|c|c|c|c|c|c|c|c|c|c|}
\hline & & \multirow[t]{2}{*}{ W A } & \multirow{2}{*}{$\begin{array}{l}\text { D } 1 \\
5 \cdot 8 \\
-5 \cdot 3\end{array}$} & \multirow[t]{2}{*}{ L 1} & \multicolumn{5}{|c|}{ D 2} & \multirow[b]{2}{*}{$\begin{array}{l}5 \cdot 9 \\
-5 \cdot 6\end{array}$} & & \multirow[t]{2}{*}{ L 2} & \multirow[t]{2}{*}{$\mathbf{R} \mathbf{N}$} \\
\hline & & & & & $\begin{array}{l}2 \cdot 2 \\
-3 \cdot 8\end{array}$ & $\begin{array}{l}3.6 \\
-3.3\end{array}$ & $\begin{array}{l}4 \cdot 8 \\
-4 \cdot 7\end{array}$ & $\begin{array}{l}4 \cdot 6 \\
-4 \cdot 5\end{array}$ & $\begin{array}{l}4 \cdot 2 \\
-4 \cdot 0\end{array}$ & & $5 \cdot 5$ & & \\
\hline $1 / 4$ & $\begin{array}{l}\text { MR } \\
\text { RM } \\
\text { ML } \\
\text { LM } \\
\text { mean } \\
\delta\end{array}$ & $\begin{array}{r}+139 \\
-129 \\
-106 \\
+234 \\
152 \\
-82 \\
\end{array}$ & $\begin{array}{r}119 \\
82 \\
62 \\
135 \\
99 \\
+28 \\
\end{array}$ & $\begin{array}{r}145 \\
79 \\
30 \\
165 \\
105 \\
+22\end{array}$ & $\begin{array}{r}191 \\
139 \\
82 \\
221 \\
158 \\
+48 \\
\end{array}$ & $\begin{array}{r}178 \\
178 \\
95 \\
181 \\
158 \\
+21\end{array}$ & $\begin{array}{r}142 \\
125 \\
40 \\
122 \\
107 \\
+25\end{array}$ & $\begin{array}{r}99 \\
63 \\
36 \\
109 \\
77 \\
+27\end{array}$ & $\begin{array}{r}73 \\
69 \\
16 \\
96 \\
63 \\
+21 \\
\end{array}$ & $\begin{array}{r}56 \\
106 \\
23 \\
66 \\
62 \\
-2\end{array}$ & $\begin{array}{r}53 \\
102 \\
0 \\
73 \\
57 \\
+6\end{array}$ & $\begin{array}{r}165 \\
148 \\
49 \\
145 \\
127 \\
+3\end{array}$ & $\begin{array}{r}191 \\
149 \\
92 \\
191 \\
156 \\
+35\end{array}$ \\
\hline $3 / 4$ & $\begin{array}{l}\text { MR } \\
\text { RM } \\
\text { ML } \\
\text { LM } \\
\text { mean } \\
\delta\end{array}$ & $\begin{array}{r}-168 \\
+254 \\
+280 \\
-264 \\
241 \\
+25\end{array}$ & $\begin{array}{r}158 \\
191 \\
208 \\
235 \\
198 \\
+1\end{array}$ & $\begin{array}{r}139 \\
241 \\
211 \\
238 \\
207 \\
+18\end{array}$ & $\begin{array}{r}277 \\
333 \\
254 \\
287 \\
288 \\
+6 \\
\end{array}$ & $\begin{array}{r}251 \\
301 \\
280 \\
261 \\
273 \\
+17\end{array}$ & $\begin{array}{r}184 \\
195 \\
195 \\
247 \\
205 \\
-10\end{array}$ & $\begin{array}{l}125 \\
198 \\
158 \\
224 \\
173 \\
+2\end{array}$ & $\begin{array}{l}109 \\
135 \\
122 \\
188 \\
138 \\
-9\end{array}$ & $\begin{array}{r}72 \\
165 \\
132 \\
221 \\
148 \\
+1\end{array}$ & $\begin{array}{r}63 \\
132 \\
125 \\
211 \\
132 \\
-4\end{array}$ & $\begin{array}{l}165 \\
251 \\
185 \\
247 \\
212 \\
+6\end{array}$ & $\begin{array}{r}264 \\
370 \\
287 \\
307 \\
307 \\
+21\end{array}$ \\
\hline$\frac{1 / 4+3 / 4}{2}$ & $\delta^{M}$ & $\begin{array}{r}196 \\
16\end{array}$ & $\begin{array}{r}148 \\
27\end{array}$ & $\begin{array}{r}156 \\
4\end{array}$ & $\begin{array}{r}223 \\
42\end{array}$ & $\begin{array}{r}215 \\
4\end{array}$ & $\begin{array}{r}156 \\
35\end{array}$ & $\begin{array}{r}125 \\
25\end{array}$ & $\begin{array}{r}100 \\
30\end{array}$ & $\begin{array}{r}105 \\
1\end{array}$ & $\begin{array}{l}95 \\
10\end{array}$ & $\begin{array}{r}169 \\
3\end{array}$ & $\begin{array}{r}231 \\
14\end{array}$ \\
\hline $1 / 3$ & $\begin{array}{l}\text { MR } \\
\text { RM } \\
\text { ML } \\
\text { LM } \\
\text { mean } \\
\delta\end{array}$ & $\begin{array}{r}+290 \\
-260 \\
-303 \\
+297 \\
288 \\
+6\end{array}$ & $\begin{array}{r}274 \\
231 \\
274 \\
271 \\
262 \\
+10\end{array}$ & $\begin{array}{l}254 \\
257 \\
274 \\
288 \\
278 \\
+2\end{array}$ & $\begin{array}{r}323 \\
271 \\
297 \\
287 \\
294 \\
+11\end{array}$ & $\begin{array}{l}304 \\
297 \\
297 \\
271 \\
292 \\
-5\end{array}$ & $\begin{array}{r}284 \\
251 \\
261 \\
270 \\
266 \\
+10\end{array}$ & $\begin{array}{r}228 \\
224 \\
221 \\
264 \\
234 \\
+12\end{array}$ & $\begin{array}{l}185 \\
200 \\
165 \\
208 \\
189 \\
+7\end{array}$ & $\begin{array}{l}172 \\
241 \\
148 \\
251 \\
203 \\
+8\end{array}$ & $\begin{array}{l}158 \\
214 \\
165 \\
251 \\
172 \\
+7\end{array}$ & $\begin{array}{r}205 \\
284 \\
238 \\
257 \\
246 \\
+15\end{array}$ & $\begin{array}{r}316 \\
312 \\
288 \\
296 \\
328 \\
+28\end{array}$ \\
\hline $2 / 4$ & MR & 82 & 96 & 30 & 79 & 99 & 46 & 90 & 0 & 0 & 0 & 92 & 33 \\
\hline
\end{tabular}




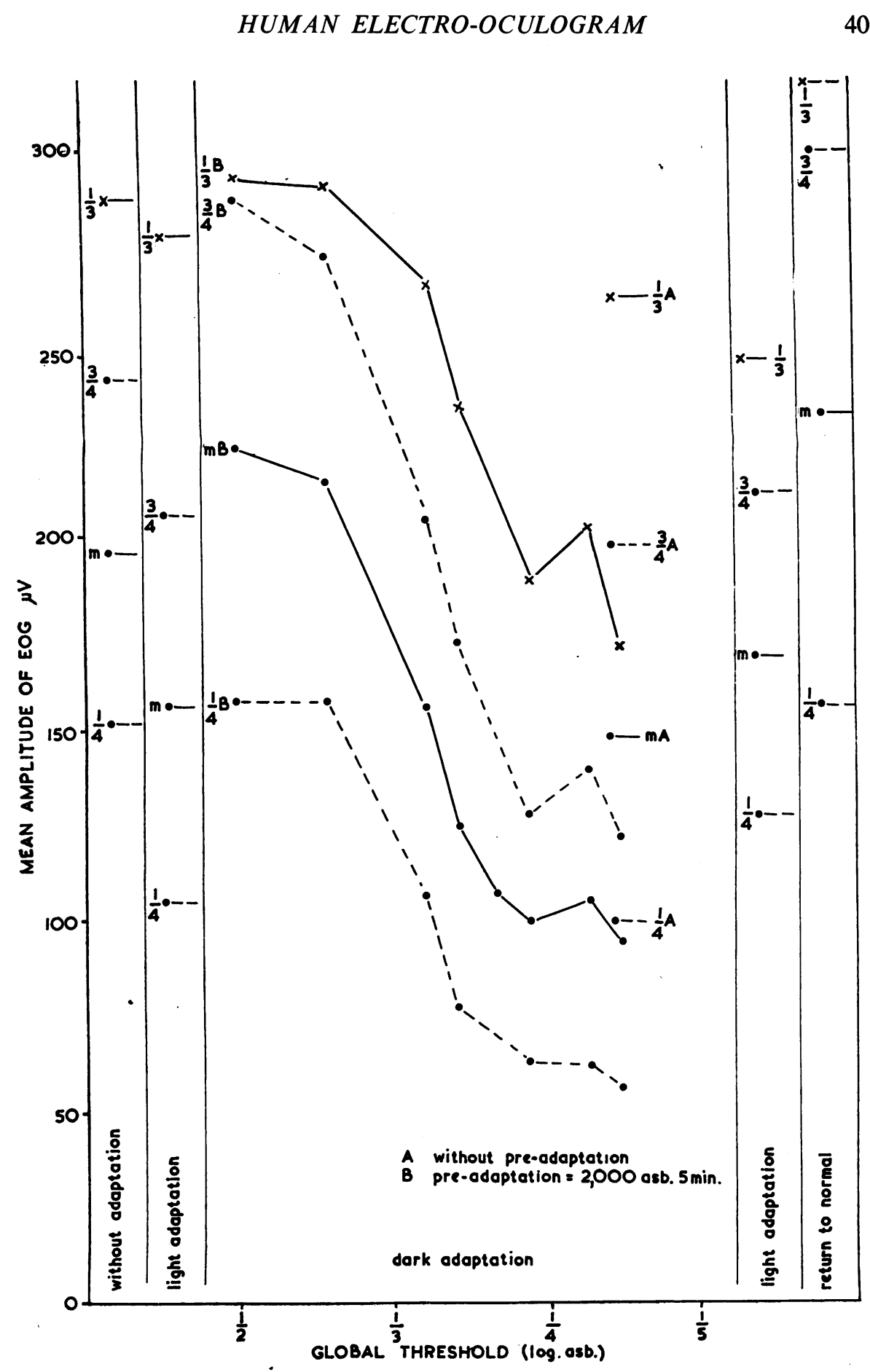

Fig. 5.-Subject 4. 


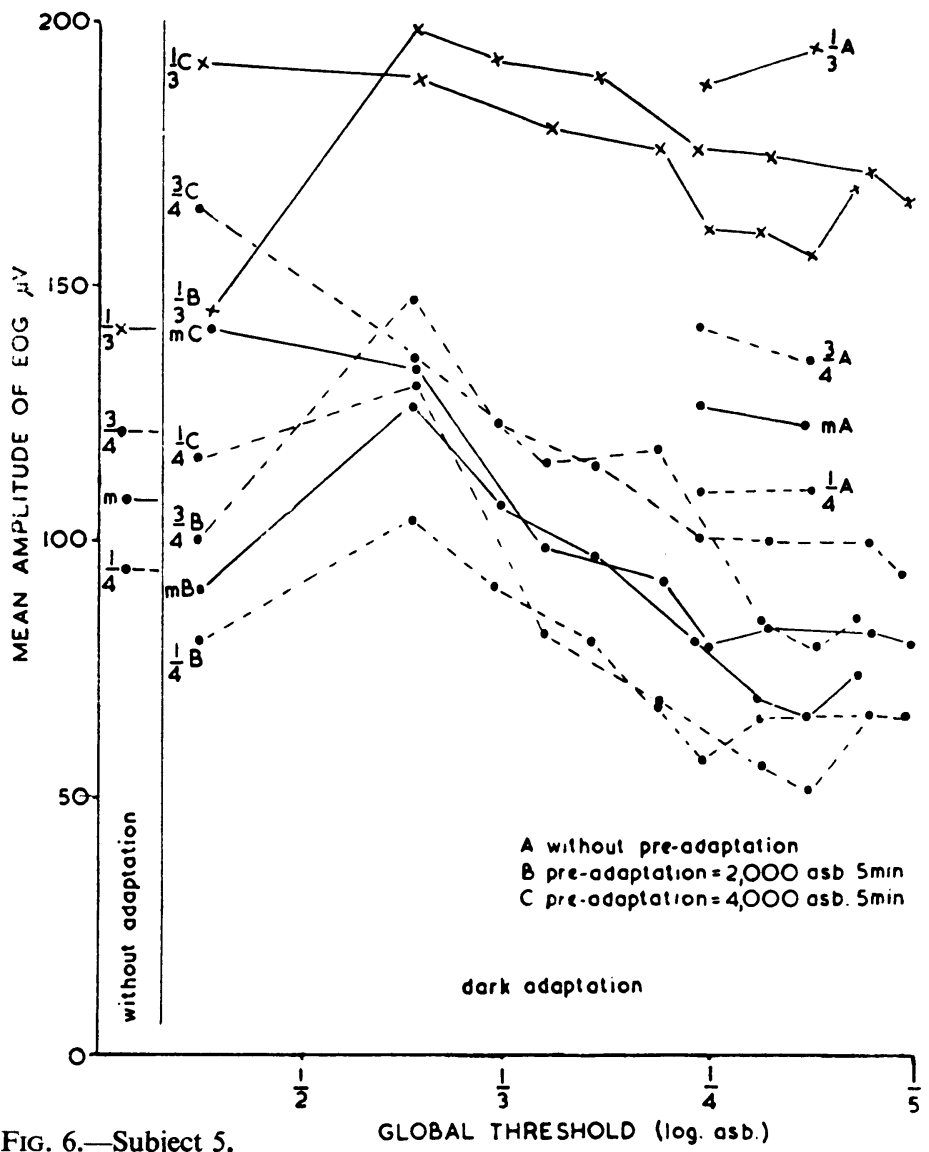

Fig. 6.-Subject 5.

TABLE V

\begin{tabular}{|c|c|c|c|c|c|c|c|c|c|}
\hline & & \multirow[t]{2}{*}{$\mathbf{W} \mathbf{A}$} & \multicolumn{2}{|c|}{ D 1} & \multicolumn{5}{|c|}{ D 2} \\
\hline & & & $4 \cdot 2-5 \cdot 9$ & $5 \cdot 6-5 \cdot 4$ & $2 \cdot 8-2 \cdot 2$ & $3 \cdot 6-3 \cdot 3$ & $3 \cdot 1-3 \cdot 0$ & $4 \cdot 6-4 \cdot 5$ & $4 \cdot 2-5 \cdot 9$ \\
\hline $1 / 4$ & $\begin{array}{l}\text { MR } \\
R M \\
\text { ML } \\
\text { LM } \\
\text { mean } \\
\delta \\
\end{array}$ & $\begin{array}{r}+110 \\
-93 \\
-50 \\
+123 \\
93 \\
+28 \\
\end{array}$ & $\begin{array}{r}129 \\
96 \\
89 \\
116 \\
108 \\
+15 \\
\end{array}$ & $\begin{array}{r}129 \\
108 \\
78 \\
120 \\
109 \\
+16 \\
\end{array}$ & $\begin{array}{r}112 \\
80 \\
86 \\
129 \\
80 \\
+19 \\
\end{array}$ & $\begin{array}{r}110 \\
106 \\
86 \\
115 \\
104 \\
+8 \\
\end{array}$ & $\begin{array}{r}110 \\
103 \\
65 \\
83 \\
90 \\
+6 \\
\end{array}$ & $\begin{array}{r}98 \\
70 \\
63 \\
88 \\
79 \\
+13 \\
\end{array}$ & $\begin{array}{r}81 \\
63 \\
33 \\
56 \\
58 \\
+10 \\
\end{array}$ \\
\hline $3 / 4$ & $\begin{array}{l}\text { MR } \\
\text { RM } \\
\text { ML } \\
\text { LM } \\
\text { mean } \\
\delta\end{array}$ & $\begin{array}{r}-73 \\
+123 \\
+157 \\
-133 \\
121 \\
+18\end{array}$ & $\begin{array}{r}109 \\
166 \\
161 \\
129 \\
141 \\
+19\end{array}$ & $\begin{array}{r}100 \\
153 \\
161 \\
123 \\
134 \\
+22\end{array}$ & $\begin{array}{l}121 \\
150 \\
163 \\
129 \\
100 \\
+8\end{array}$ & $\begin{array}{r}131 \\
153 \\
166 \\
129 \\
145 \\
+15\end{array}$ & $\begin{array}{r}100 \\
117 \\
156 \\
117 \\
122 \\
+14\end{array}$ & $\begin{array}{r}86 \\
108 \\
150 \\
112 \\
113 \\
+15\end{array}$ & $\begin{array}{r}65 \\
93 \\
129 \\
115 \\
100 \\
+11\end{array}$ \\
\hline$\frac{1 / 4+3 / 4}{2}$ & $\delta^{M}$ & $\begin{array}{r}108 \\
10\end{array}$ & $\begin{array}{r}125 \\
4\end{array}$ & $\begin{array}{r}121 \\
6\end{array}$ & $\begin{array}{l}90 \\
11\end{array}$ & $\begin{array}{r}125 \\
7\end{array}$ & $\begin{array}{r}106 \\
8\end{array}$ & $\begin{array}{r}96 \\
2\end{array}$ & $\begin{array}{r}79 \\
2\end{array}$ \\
\hline $1 / 3$ & $\begin{array}{l}\text { MR } \\
\text { RM } \\
\text { ML } \\
\text { LM } \\
\text { mean } \\
\delta\end{array}$ & $\begin{array}{r}+197 \\
-166 \\
-209 \\
+189 \\
190 \\
+3\end{array}$ & $\begin{array}{l}189 \\
181 \\
211 \\
166 \\
187 \\
-9\end{array}$ & $\begin{array}{l}180 \\
191 \\
207 \\
197 \\
193 \\
-5\end{array}$ & $\begin{array}{l}198 \\
179 \\
214 \\
180 \\
139 \\
-4\end{array}$ & $\begin{array}{l}194 \\
198 \\
219 \\
179 \\
198 \\
-9\end{array}$ & $\begin{array}{r}192 \\
188 \\
214 \\
164 \\
192 \\
-11\end{array}$ & $\begin{array}{l}197 \\
177 \\
214 \\
168 \\
189 \\
-6\end{array}$ & $\begin{array}{l}170 \\
170 \\
195 \\
163 \\
174 \\
-8\end{array}$ \\
\hline $2 / 4$ & MR & 88 & 31 & 0 & 12 & 20 & 25 & 25 & 25 \\
\hline
\end{tabular}




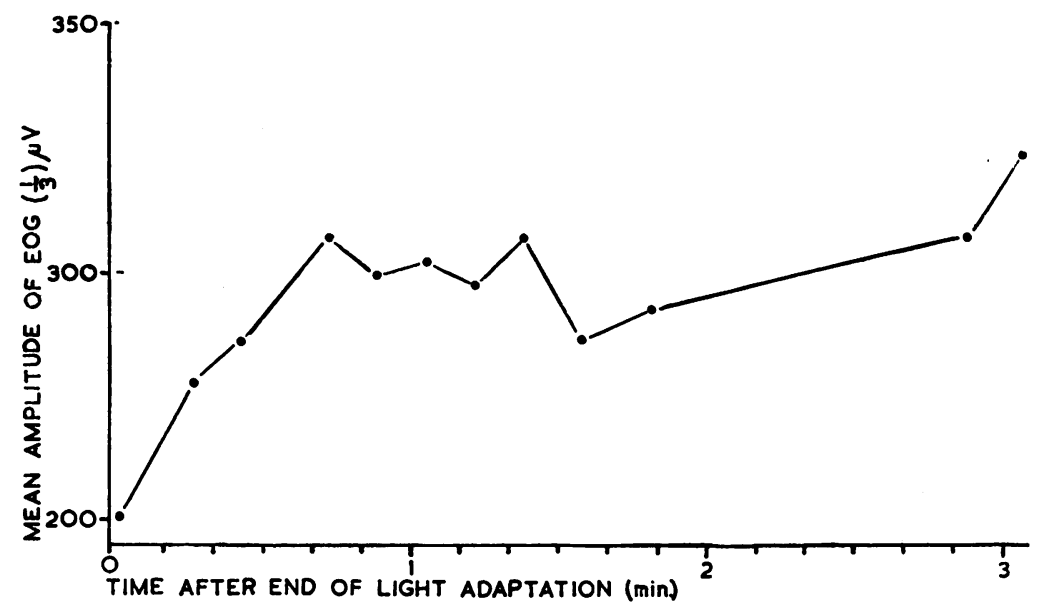

FIG. 7.- Modification of amplitude of EOG after the end of light adaptation (Subject 4).

\section{SUBJECT 5}

\begin{tabular}{|c|c|c|c|c|c|c|c|c|c|}
\hline \multirow[b]{2}{*}{$5 \cdot 8-5 \cdot 4$} & \multirow[b]{2}{*}{$5 \cdot 3-5 \cdot 1$} & \multirow[b]{2}{*}{$5 \cdot 1$} & \multicolumn{7}{|c|}{ D 3} \\
\hline & & & $2 \cdot 8-2 \cdot 2$ & $3.6-3.2$ & $4 \cdot 9-4 \cdot 7$ & $4 \cdot 3-4 \cdot 2$ & $5 \cdot 8-5 \cdot 7$ & $5 \cdot 4$ & $5 \cdot 3$ \\
\hline $\begin{array}{r}100 \\
38 \\
65 \\
61 \\
66 \\
+14\end{array}$ & $\begin{array}{r}86 \\
70 \\
36 \\
65 \\
64 \\
+11\end{array}$ & $\begin{array}{r}65 \\
70 \\
50 \\
71 \\
64 \\
+\quad 4\end{array}$ & $\begin{array}{r}156 \\
100 \\
54 \\
153 \\
116 \\
+39\end{array}$ & $\begin{array}{r}153 \\
76 \\
166 \\
119 \\
128 \\
+8\end{array}$ & $\begin{array}{r}119 \\
67 \\
55 \\
86 \\
82 \\
+21\end{array}$ & $\begin{array}{r}92 \\
55 \\
32 \\
44 \\
68 \\
+25\end{array}$ & $\begin{array}{r}70 \\
46 \\
20 \\
85 \\
55 \\
+22\end{array}$ & $\begin{array}{r}63 \\
58 \\
16 \\
66 \\
51 \\
+14\end{array}$ & $\begin{array}{r}70 \\
53 \\
53 \\
85 \\
65 \\
+12\end{array}$ \\
\hline $\begin{array}{r}60 \\
84 \\
129 \\
119 \\
98 \\
+8\end{array}$ & $\begin{array}{r}61 \\
96 \\
128 \\
106 \\
98 \\
+16\end{array}$ & $\begin{array}{r}55 \\
75 \\
129 \\
111 \\
92 \\
+22\end{array}$ & $\begin{array}{r}136 \\
192 \\
197 \\
129 \\
163 \\
+28\end{array}$ & $\begin{array}{r}119 \\
154 \\
172 \\
131 \\
144 \\
+18\end{array}$ & $\begin{array}{r}80 \\
111 \\
151 \\
119 \\
115 \\
+16\end{array}$ & $\begin{array}{r}81 \\
114 \\
136 \\
133 \\
116 \\
+9\end{array}$ & $\begin{array}{r}51 \\
78 \\
104 \\
100 \\
83 \\
+9\end{array}$ & $\begin{array}{r}50 \\
66 \\
118 \\
84 \\
79 \\
+12\end{array}$ & $\begin{array}{r}50 \\
75 \\
116 \\
86 \\
82 \\
+15\end{array}$ \\
\hline $\begin{array}{r}82 \\
6\end{array}$ & 81 & $\begin{array}{l}78 \\
18\end{array}$ & $\begin{array}{r}140 \\
11\end{array}$ & $\begin{array}{r}136 \\
10\end{array}$ & $\begin{array}{r}98 \\
5\end{array}$ & $\begin{array}{l}92 \\
16 \\
\end{array}$ & $\begin{array}{l}69 \\
12 \\
\end{array}$ & $\begin{array}{r}65 \\
2 \\
\end{array}$ & $\begin{array}{r}73 \\
0 \\
\end{array}$ \\
\hline $\begin{array}{r}166 \\
153 \\
195 \\
181 \\
173 \\
0\end{array}$ & $\begin{array}{r}164 \\
166 \\
200 \\
154 \\
170 \\
-12\end{array}$ & $\begin{array}{r}146 \\
154 \\
190 \\
166 \\
164 \\
-8\end{array}$ & $\begin{array}{r}186 \\
189 \\
230 \\
166 \\
192 \\
-17\end{array}$ & $\begin{array}{l}207 \\
163 \\
214 \\
166 \\
188 \\
-1\end{array}$ & $\begin{array}{l}179 \\
177 \\
198 \\
163 \\
179 \\
-8\end{array}$ & $\begin{array}{l}163 \\
169 \\
191 \\
171 \\
176 \\
-6\end{array}$ & $\begin{array}{l}154 \\
148 \\
167 \\
166 \\
158 \\
+1\end{array}$ & $\begin{array}{l}144 \\
146 \\
169 \\
158 \\
154 \\
-3\end{array}$ & $\begin{array}{l}154 \\
143 \\
181 \\
158 \\
159 \\
-3\end{array}$ \\
\hline 25 & 25 & 80 & 32 & 16 & 16 & 0 & 0 & 0 & 0 \\
\hline
\end{tabular}


EOG; since it is not possible to measure a true perception threshold without removing the adapting surrounding light, the study of the possible relation between the value of the resting potential and the threshold by means of EOG encounters insurmountable difficulties which are certainly partly due to noncontrollable psychogalvanic reflexes.

(5) It is recommended that the EOG should be used clinically in testing the motility of the eyes or as a functional test of the retina; it should be used in moderate brightness, without previous dark or light adaptation.

\section{REFERENCES}

Kohlrausch, A. (1931). "Elektrische Erscheinungen am Auge", In A. Bethe, G. von BERGMANN, G. EMBDEN, and A. Ellinger, "Handbuch der normalen und pathologischen Physiologie ", vol. 12, pt. 2, pp. 1393-1496. Springer, Berlin.

MARG, E. (1951). Arch. Ophthal. (Chicago), 45, 169-185.

Miles, W. R. (1940). Science, 91, 456.

WulfF, V. J., and FreYbURGER, S. W. (1948). Anat. Rec., 101, 665. 